\title{
Studies on the Mechanism of the Volatile Oils from Caoguo-4 Decoction in Regulating Spleen Deficiency Diarrhea by Adjusting Intestinal Microbiota
}

\author{
Li Mei, ${ }^{1,2}$ Fang Wang $\mathbb{D},{ }^{1}$ Ming Yang, ${ }^{1}$ Zhiyong Liu $\left(\mathbb{D},{ }^{1}\right.$ Liangfeng Wang, ${ }^{1,3}$ Qingyao Chen, ${ }^{1}$ \\ Fengqin $\mathrm{Li}^{1}{ }^{1}$ and Xiaofei Zhang ${ }^{10}{ }^{1,4}$
}

${ }^{1}$ Jiangxi University of Chinese Medicine, 1688 Meiling Avenue, Wanli District, Nanchang 330004, China

${ }^{2}$ Inner Mongolia Minzu University, No. 536 West Huolinhe Street, Horqin District,

Tongliao Inner Mongolia Autonomous Region 028000, China

${ }^{3}$ College of Pharmacy, Shanghai University of Chinese Medicine, Shanghai 201203, China

${ }^{4}$ Shaanxi University of Chinese Medicine, Xixian Avenue, Xixian District, Xianyang 712046, China

Correspondence should be addressed to Fang Wang; wangfang09421@126.com and Xiaofei Zhang; 84964547@qq.com

Received 7 October 2021; Accepted 13 December 2021; Published 27 January 2022

Academic Editor: Wuquan Deng

Copyright (C) $2022 \mathrm{Li}$ Mei et al. This is an open access article distributed under the Creative Commons Attribution License, which permits unrestricted use, distribution, and reproduction in any medium, provided the original work is properly cited.

Background. The Caoguo-4 decoction, a classical Mongolian medicine formula, is widely used to treat spleen deficiency diarrhea (SDD) in Mongolian for decades. Previously, the Caoguo-4 decoction volatile oil has been confirmed to be effective in ameliorating symptoms of spleen deficiency diarrhea in an animal model. However, the underlying mechanism of the Caoguo4 decoction volatile oil is yet to be established. The aim of the current study was to investigate the antidiarrheal effects and mechanism of the Caoguo-4 decoction volatile oil. Method. Wistar rats were randomly divided into 5 groups of 10 animals including control, model, positive, Caoguo-4 decoction, and Caoguo-4 decoction volatile oil groups (10 rats in each group). All the rats, besides those in the control group, were induced to develop SDD by a bitter-cold purgation method with Xiaochengqi decoction. The antidiarrheal effect of Caoguo-4 decoction volatile oil was evaluated by pathological section, serum D-xylose and AMS content, plasma MTL content, and gut microbiota analysis via $16 \mathrm{~S}$ rRNA sequencing. Results. The results showed that the developed SDD rat model (model group) had decreased food intake, increased weight loss, soft stool, and bad hair color. When compared with the control group, serum was significantly reduced serum D-xylose and AML but increased MTL levels in the model group $(p<0.05)$. However, after treatment with either the Caoguo- 4 decoction (the decoction group) or Smecta (the positive group) or volatile oil from the Caoguo- 4 decoction (the volatile oil group), a significant increase in the serum D-xylose levels was observed. Additionally, AML levels significantly increased in the positive and volatile oil groups, and MTL levels significantly decreased in the decoction and volatile oil groups, when compared with the model group $(p<0.05)$. The pathological changes of the intestinal mucosa showed that the structure of the epithelium in the villi of the small intestine was affected, deformed, and incomplete in the model group when compared with the control group. However, either the decoction group or the volatile oil group recovered the villous morphology. The results of OTU analysis and alpha diversity analysis of intestinal bacteria showed that the intestinal microbiota of the SDD model rats showed an obvious decrease in richness and diversity of intestinal microbiota. But the intervention treatment of decoction and volatile oil could significantly recover the richness and diversity of intestinal microbiota. Conclusion. The intestinal microbiota destroyed in SDD modelling could be significantly improved by the Caoguo- 4 decoction volatile oils, which provides reference for clinical medication.

\section{Introduction}

Spleen deficiency diarrhea (SDD) is a common disease of traditional Chinese medicine (TCM). The main cause of
SDD according to TCM is considered to be spleen deficiency, endophytic dampness, Qi consumption, and stomach disharmony, resulting in conductive dysfunction of the intestines $[1,2]$. The deficient spleen fails to transport and the weak 


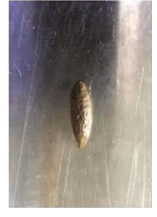

CG

(a)

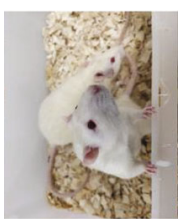

CG

(d)



MG

(b)

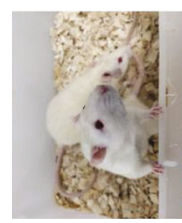

CG

(e)

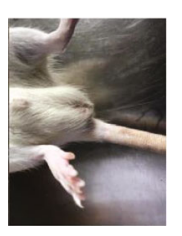

CG

(c)



MG

(f)

FIGURE 1: Microcosmic indexes of rats in the control group and the model group. Note: CG: control group; MG: model group: (a) stool from the control group; (b) stool from the model group; (c) anal area of the control group; (d) anal area of the model group; (e) posture of the control group; (f) posture of the model group.

stomach fails to digest and process foods; thus, the water and foods are then retained to affect the ascent of spleen yang; consequently, it collapses to cause diarrhea [3]. At present, the specific pathogenesis of SDD remains unknown: immune dysfunction, intestinal barrier dysfunction, mental factors, etc. [4]. With the deepening of research, gut microbiota has become one of the most critical factors in SDD progression, accompanied by diarrhea induced by dysfunctional or infection intestinal environment $[5,6]$. Related research also confirmed that the quantity of beneficial physiological bacteria such as Lactobacillus and Bifidobacterium in the intestine of mice is negatively correlated with the severity of the symptoms of SDD in mice; that is, the more severe the symptom of SDD in mice, the smaller the quantity of Lactobacillus and Bifidobacterium in the intestine of mice, leading to disorder of gut microbiota [7]. Therefore, a better understanding of the gastrointestinal microflora in SDD has been of great significance. Acupoint application therapy is the application of Chinese herbal medicine to the corresponding points to regulate meridians, yin and yang, and qi as well as the blood of the human body [8]. The Shenque point has a thin cuticle making it conducive to rapid drug absorption, and its location close to the intestinal tract strengthens the spleen and stomach by promoting the flow of Qi and relaxing the bowels [9]. Increasing evidence suggests that TCM achieved a good therapeutic effect for SDD for its less toxicity and equal or even better antidiarrheal efficacy [10]. Caoguo-4 decoction is a kind of traditional Mongolian medicine formula, originated from the medical classic of "Yi Fang Feng Ji." It has long been used clinically for the maintenance of "Heyi" and upward "Heyi" disease and specifically has been identified to have a significant effect on spleen deficiency diarrhea. "Mongolian Medicine Prescription" [11]: "Used for the treatment of stomach "Heyi "syndrome, head tingling, spleen disease, etc." "Chinese Medical Encyclopedia (Mongolian version)" recorded [12]: "Indications for head stabbing pain, abdominal distension and bowel rumbling caused by Ascending "Heyi" and "Siming Heyi." "It is composed of Amomum tsao-ko Crevost et Lemarie, Eugenia caryophyllata
TABLE 1: The contents of D-xylose, AML, and MTL in blood of rats with spleen deficiency diarrhea.

\begin{tabular}{lccc}
\hline Group & D-Xylose & AMS & MTL \\
\hline CG & $1.66 \pm 0.30$ & $4294 \pm 848$ & $167.58 \pm 25.73$ \\
MG & $0.81 \pm 0.37^{*}$ & $2469 \pm 929^{*}$ & $196.34 \pm 23.47^{*}$ \\
TG & $2.52 \pm 1.20^{\#}$ & $2880 \pm 371$ & $160.47 \pm 28.68^{\#}$ \\
HG & $2.15 \pm 0.41^{\# \#}$ & $3862 \pm 559^{\#}$ & $145.20 \pm 17.99^{\# \#}$ \\
YXG & $2.07 \pm 0.54^{\# \#}$ & $3712 \pm 740^{\#}$ & $202.03 \pm 62.39$ \\
\hline
\end{tabular}

Note: CG: control group; MG: model group; TG: soup group; HG: oil group; YXG: positive group. Compared with the control group, ${ }^{*} p<0.05,{ }^{* *} p<$ 0.01 ; compared with the model group, ${ }^{\#} p<0.05,{ }^{\# \#} p<0.01$.

Thunb., Aucklandia lappa Decne., and Foeniculum vulgare Mill., which all belong to the "warm-natured" category of the Mongolian medicine, with a role specifically in dispelling the spleen and stomach chill. However, Caoguo- 4 decoction has a curative clinical effect on SDD, but with limited development due to being short of defined pharmacodynamics and mechanisms.

Volatile oils refer to the active components from TCM. Valuable properties of volatile oils have been confirmed experimentally or empirically. Examples include [13] Eugenia caryophyllata Thunb. (strengthening the spleen and resisting bacteria), Aucklandia lappa Decne. (moving Qi and relieving pain), Amomum tsao-ko Crevost et Lemarie (invigorating the stomach and dispelling dampness), Foeniculum vulgare Mill. (regulating Qi flowing for strengthening the spleen), etc. In the previous study from this group, the Caoguo-4 decoction volatile oil has been confirmed to be effective in ameliorating symptoms of the SDD rat model, which is comparable to the effect of the Caoguo-4 decoction. However, the underlying mechanisms by which the Caoguo4 decoction volatile oil exhibits antidiarrheal activity remains poorly understood. Hence, the aim of this study was to investigate the antidiarrheal effect and mechanism of the Caoguo-4 decoction volatile oil through regulation of intestinal microbiota. 


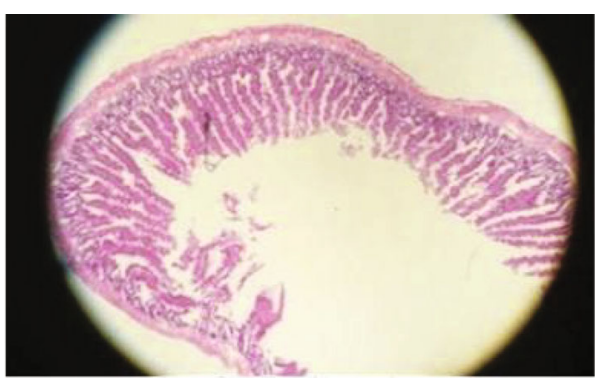

(a)

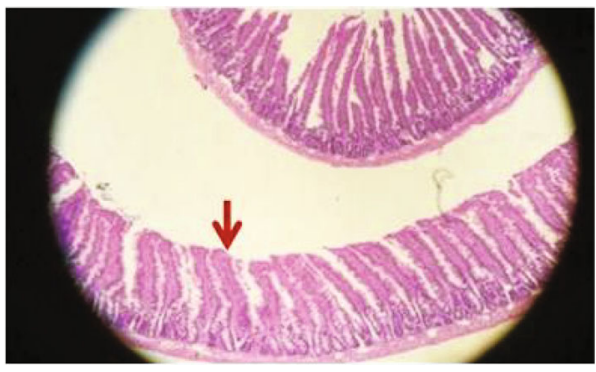

(c)

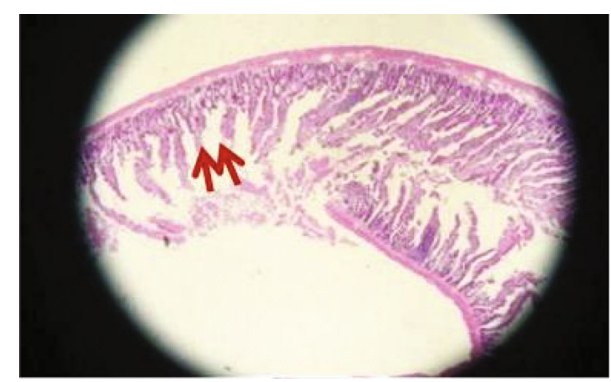

(b)

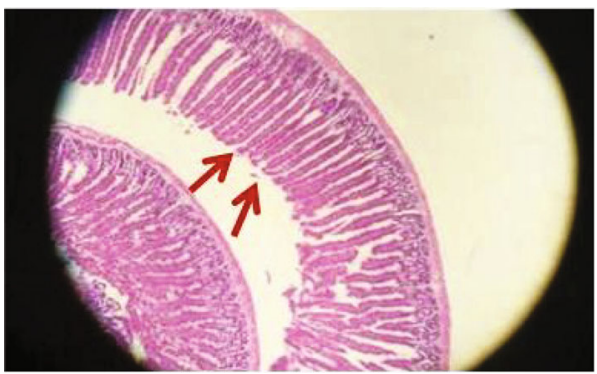

(d)

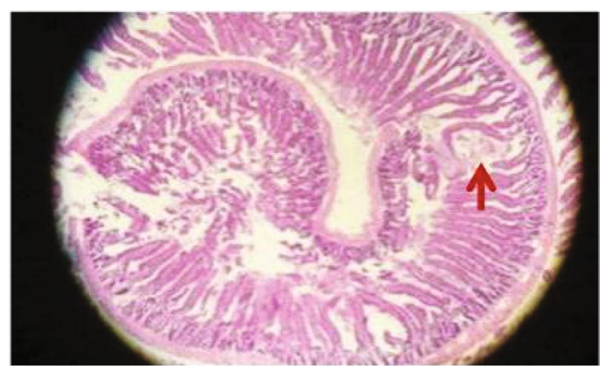

(e)

Figure 2: Effect of each group on small intestinal mucosa histopathology in rats with spleen deficiency diarrhea (hematoxylin-eosin; 40x magnification): (a) CG group, (b) MG group, (c) TG group, (d) HG group, and (e) YXG group.

\section{Materials and Methods}

2.1. Materials and Chemicals. Amomum tsao-ko (batch number: 1709009), cloves (batch number: 1710004), fennel (batch number: 1711009), Costustoot (batch number: 1703003), Rheum officinale (batch number: 1806007), fructus aurantii immaturus (batch number: 1806008), and Magnolia officinalis (batch number: 1805003) were purchased from Jiangxi Zhangshu Tianqitang Chinese Herbal Slices Co., Ltd. (Jiangxi, China). The commercial enzyme-linked immunosorbent assay (ELISA) kits for D-xylose, serum motilin (MTL), and amylase were purchased from Jiancheng Enzymatic Immunity Co., Ltd. (Nanjing, China). Jojoba oil was purchased from Dimei Biotechnology Co., Ltd. (Guangzhou, China).

2.2. Plant Materials and Volatile Oil Extraction. Caoguo-4 decoction, a kind of traditional Mongolian medicine formula, contains the following ingredient: Amomum tsao-ko Crevost et Lemarie (also known as CaoGuo in China), Eugenia caryophyllata Thunb. (also known as DingXiang in China), Aucklandia lappa Decne. (also known as MuXiang in China), and Foeniculum vulgare Mill. (also known as
HuiXiang in China). All the herbs were purchased from Chengdu Huichu Technology Co., Ltd. (Chengdu, China), which were authenticated by Professor Fei Ge (Jiangxi University of Traditional Chinese Medicine, Nanchang, China). According to the ratio of CG : MX : DX : HX $=5: 5: 3: 3$, the dried Chinese herbs were crushed into coarse powder.

Caoguo-4 decoction was prepared as follows: $100 \mathrm{~g}$ crude Caoguo-4 decoction was made into powder and put in 10 times of water for 15 minutes, boiled for 30 minutes, filtered, and collected. Repeat such procedures. Puted the two liquid together, concentrated them to $0.256 \mathrm{~g} / \mathrm{ml}$.

Caoguo-4 decoction volatile oil was extracted by the steam distillation method. The steam distillations were carried out according to the Chinese Pharmacopoeia (2020). Briefly, $360 \mathrm{~g}$ of Caoguo-4 decoction medicinal powder was completely immersed in Milli-Q water in proportion of $1: 10(W / V)$ for $30 \mathrm{~min}$. The mixture was submitted to hydrodistillation in a Clevenger-type apparatus at temperature of $100^{\circ} \mathrm{C}$ for 240 minutes. Volatile oil was dried over $\mathrm{Na}_{2} \mathrm{SO}_{4}$. The yield of volatile oil was $5 \%(V / W)$, and the oil was stored at $-20^{\circ} \mathrm{C}$ until analysis. For animal experiment, the volatile oil was diluted with jojoba oil with the dilution ratio of $1: 10(\mathrm{~V} / \mathrm{V})$. 


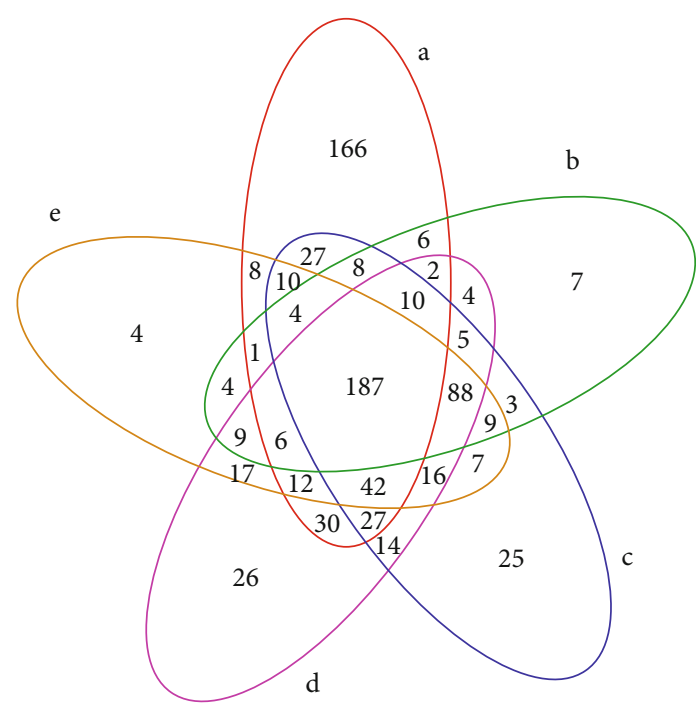

Figure 3: OTU Venn figure: $\mathrm{a}-\mathrm{CG}$ group, $\mathrm{b}-\mathrm{MG}$ group, $\mathrm{c}-\mathrm{TG}$ group, $\mathrm{d}-\mathrm{HG}$ group, and $\mathrm{e}-\mathrm{YXG}$ group.



FIGURE 4: PCA based on OTU level: a-CG group, b-MG group, c-TG group, d-HG group, and e-YXG group.

\subsection{Animal Experimental Design}

2.3.1. Animal. SPF Wistar rats weighing $\sim 200-220 \mathrm{~g}$ were purchased from Lai-Ke-Jing-Da Experimental Animal Co. Ltd. (Hunan, China) and consisted of both genders at a proportion of $1: 1$. The animals were housed individually in the Experimental Animal Center of Jiangxi University of Chinese Medicine (Jiangxi, China). Standard tap water and rat food were available. After acclimation for 7 days, all rats were randomly divided into 5 groups of 10 animals each as follows: the normal control group (CG), the spleen deficiency diarrhea model group of treatment groups (MG), the positive group of treatment groups (YXG), the Caoguo4 decoction group of treatment groups (TG), and the Caoguo-4 decoction volatile oil group of treatment groups (HG). The spleen deficiency diarrhea model was induced


FIgURe 5: Alpha diversity index box figure: a-CG group, $\mathrm{b}-\mathrm{MG}$ group, c-TG group, d-HG group, and e-YXG group.

in the MG group, the YXG group, the TG group, and the HG group.

During all experiments, water and food were available ad libitum. All animal procedures were conducted in accordance with the institutional guidelines approved by the Ethics Committee of the Jiangxi University of Chinese Medicine (no. JZLLSC2018-0086).

2.3.2. Spleen Deficiency Diarrhea Model and Group Treatments. The spleen deficiency diarrhea (SDD) model was established using a bitter-cold purgation method with Xiaochengqi decoction $[14,15]$. Briefly, the rats were intragastrically administered with $3 \mathrm{ml} / 100 \mathrm{~g}$ Xiaochengqi decoction for 21 days once every other day. Meanwhile, the rats were not fed with any chow but drinking water freely on gavage day and were fed ad libitum on the next day. The CG group was intragastrically administered with the same volume of double-distilled water with food and water ad libitum. The YXG group was intragastrically administered with Smecta, prepared in $40 \mathrm{mg} / \mathrm{ml}$ suspension with distilled water (equivalent to 10 times the clinical dosage). The TG group was given the Caoguo-4 decoction with a mass concentration of $0.256 \mathrm{~g} / \mathrm{ml}$ (the amount of $10 \mathrm{ml} / \mathrm{kg} /$ day was applied). The HG group was given the Caoguo- 4 decoction volatile oil through the umbilical cord and stimulating the Shenque acupoint with $1.92 \mathrm{ml}$ (the yield of volatile oil was $5 \%(V / W), 10$ times of the oral dose). The modelling was 
TABLE 2: Comparison of OTUs and diversity of samples between groups.

\begin{tabular}{lccccc}
\hline Groups & $A$ & Observed species & PD-whole tree & $B$ & Recovery rate \\
\hline CG & $449.86 \pm 28.72$ & $351.50 \pm 10.64$ & $34.48 \pm 0.31$ & $4.47 \pm 0.43$ & $>0.99$ \\
MG & $286.88 \pm 22.05^{* *}$ & $233.80 \pm 13.62^{* *}$ & $24.40 \pm 1.89^{* *}$ & $4.59 \pm 0.15$ & $>0.99$ \\
TG & $393.16 \pm 36.37^{\# \#}$ & $311.08 \pm 34.21^{\# \#}$ & $30.42 \pm 3.02^{\#}$ & $5.03 \pm 0.47$ & $>0.99$ \\
HG & $379.11 \pm 58.53^{\#}$ & $312.05 \pm 55.76^{\#}$ & $30.79 \pm 4.18^{\#}$ & $4.47 \pm 0.89$ & $>0.99$ \\
YXG & $337.04 \pm 61.82$ & $274.28 \pm 39.38$ & $28.17 \pm 3.04$ & $4.57 \pm 0.42$ & $>0.99$ \\
\hline
\end{tabular}

Note: CG: control group; MG: model group; TG: soup group; HG: oil group; YXG: positive group. Compared with the control group, ${ }^{*} p<0.05,{ }^{* *} p<0.01$; compared with the model group, ${ }^{\#} p<0.05,{ }^{\#} p<0.01$.

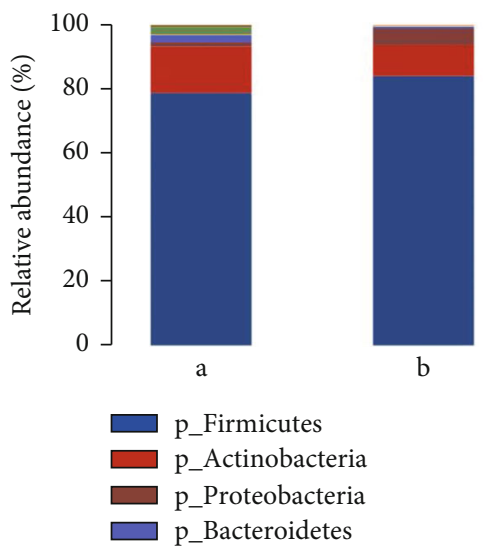

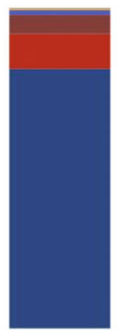

C

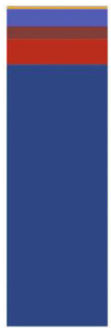

d

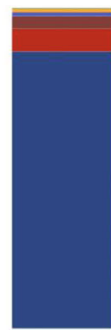

e

Figure 6: Profiling histograms of species at different groups of phylum classification levels. The horizontal coordinate is the sample name, and the longitudinal coordinates are the relative abundance of the species in the sample: $\mathrm{a}-\mathrm{CG}$ group, $\mathrm{b}-\mathrm{MG}$ group, $\mathrm{c}-\mathrm{TG}$ group, $\mathrm{d}-\mathrm{HG}$ group, and e-YXG group.

TABLE 3: Species at different groups of phylum classification levels (\%).

\begin{tabular}{lccccc}
\hline Phylum & $\begin{array}{c}\mathrm{CG} \\
\text { group }\end{array}$ & $\begin{array}{c}\mathrm{MG} \\
\text { group }\end{array}$ & $\begin{array}{c}\text { TG } \\
\text { group }\end{array}$ & $\begin{array}{c}\mathrm{HG} \\
\text { group }\end{array}$ & $\begin{array}{c}\text { YXY } \\
\text { group }\end{array}$ \\
\hline Cyanobacteria & $0.12 \%$ & $0.00 \%{ }^{* *}$ & $0.03 \%$ & $0.01 \%$ & $0.01 \%$ \\
Saccharibacteria & $2.30 \%$ & $0.00 \%^{*}$ & $0.10 \%^{\#}$ & $0.07 \%{ }^{*}$ & $0.14 \%$ \\
Proteobacteria & $1.36 \%$ & $5.24 \%{ }^{* *}$ & $5.89 \%$ & $3.97 \%{ }^{\#}$ & $3.96 \%$ \\
Tenericutes & $0.42 \%$ & $0.05 \%{ }^{* *}$ & $0.10 \%$ & $0.15 \%$ & $0.09 \%$ \\
Bacteroidetes & $2.17 \%$ & $0.64 \%$ & $1.57 \%$ & $5.47 \%$ & $1.24 \%$ \\
Firmicutes & $78.76 \%$ & $84.05 \%$ & $80.95 \%$ & $81.78 \%$ & $86.42 \%$ \\
Euryarchaeota & $0.04 \%$ & $0.00 \%{ }^{*}$ & $0.01 \%$ & $0.01 \%$ & $0.01 \%$ \\
Verrucomicrobia & $0.21 \%$ & $0.37 \%$ & $0.36 \%$ & $0.64 \%$ & $1.09 \%$ \\
Actinobacteria & $14.54 \%$ & $9.61 \%$ & $10.96 \%$ & $7.90 \%$ & $7.03 \%$ \\
Other & $0.07 \%$ & $0.03 \%$ & $0.02 \%$ & $0.02 \%$ & $0.01 \%$ \\
\hline
\end{tabular}

Note: compared with the control group, ${ }^{*} p<0.05,{ }^{* *} p<0.01$; compared with the model group, ${ }^{\#} p<0.05,{ }^{\# \#} p<0.01$.

considered successful if the following criteria were meet: (a) slow weight gain or weight loss, (b) less eating amount and more drinking volume, (c) loose stools, (d) lassitude or irritating, (e) yellow and dry hair, (f) curling up for fear of cold, and (g) D-xylose and amylase (AMS) levels measured once per week for 3 weeks.

Xiaochengqi decoction, a kind of traditional Chinese formula, contains the following ingredient: Radix et Rhizoma
Rhei, Fructus Citri aurantii immaturus, and Cortex Magnoliae officinalis with the amount ratio of $2: 3: 3$ for SDD modelling. All the herbs were purchased from Jiangzhong Chinese Medicine Yinpian Co. Ltd. (Jiangxi, China) as crude herbs. Xiaochengqi decoction was prepared as follows: the herbs were soaked in 10 times the amount of water for 30 minutes and heated for 1 hour each time, and the filtrate was combined twice and then condensed to $1 \mathrm{~g} / \mathrm{ml}$.

2.4. Sample Collection. At the $21^{\text {th }}$ day, after fasting for 24 hours, the rats were sacrificed by intraperitoneal injection of $1 \%$ pentobarbital sodium ( $50 \mathrm{mg}$ pentobarbital/kg rat body weight). Blood was harvested from the abdominal aorta into tubes with ethylene diamine tetra acetic acid. Plasma was isolated by centrifugation at $4000 \mathrm{rpm}$ for $15 \mathrm{~min}$ at $4.0^{\circ} \mathrm{C}$. The supernatant was collected and stored at $-80^{\circ} \mathrm{C}$ for later analysis. The small intestine was put into $4 \%$ buffered formalin solution and embedded in paraffin. These sections were stained with hematoxylin and eosin (HE) and periodic acid-Schiff (PAS), respectively, in accordance with the standard procedures for histopathological analysis. The intestinal feces were taken under sterile conditions and then put into sterile tubes in liquid nitrogen for further analysis. The amounts of spleen deficiencyassociated cytokines including D-xylose, AMS, and MTL were determined using ELISA kits according to the manufacturers' instructions. 


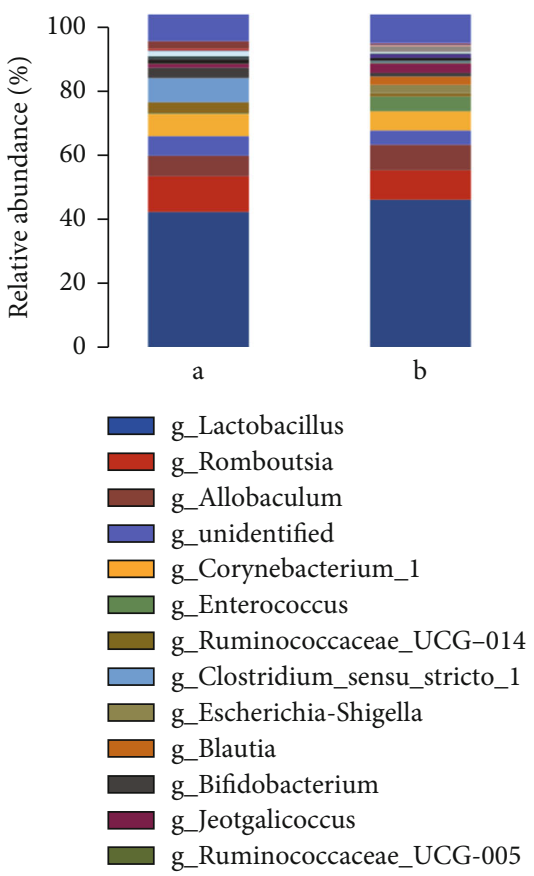

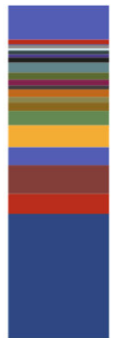

c

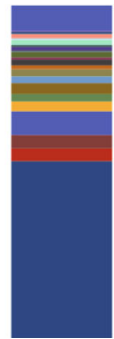

d

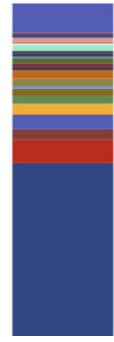

$\mathrm{e}$

$\square$ g_Subdoligranulum

g_Aerococcus

$\square$ g_Helicobacter

$\square$ g_Ruminococcaceae_UCG-013

$\square$ g_Coprococcus_2

$\square$ g_Enterorhabdus

$\square$ g_Butyricicoccus

$\square$ g_Anaerostipes

$\square$ g_Akkermansia

$\square$ g_Oligella

$\square$ g_Candidatus_Saccharimonas

$\square$ Other

Figure 7: Profiling histograms of species at different groups of genus classification levels: a-CG group, b-MG group, c-TG group, $\mathrm{d}-\mathrm{HG}$ group, and $\mathrm{e}-\mathrm{YXG}$ group.

2.5. ELISA. The rats were sacrificed by intraperitoneal injection of $1 \%$ pentobarbital sodium ( $50 \mathrm{mg}$ pentobarbital $/ \mathrm{kg}$ rat body weight). Serum was stored at $-80^{\circ} \mathrm{C}$ for analysis of Dxylose and AMS content using the enzyme-linked immunosorbent assay (D-xylose ELISA kit provided by Nanjing Jiancheng Bioengineering Institute Co., Ltd., no.20180621; AMS ELISA kit provided by Nanjing Jiancheng Bioengineering Institute Co., Ltd., no. 20180612). Plasma was stored at $-80^{\circ} \mathrm{C}$ for analysis of MTL content using the enzymelinked immunosorbent assay (MTL ELISA kit provided by Nanjing Jiancheng Bioengineering Institute Co., Ltd., no. 20180901).

2.6. Histopathological Observation. The jejunum tissues were isolated, rinsed with ice PBS, fixed in $4 \%$ paraformaldehyde solution for 24 hours, removed, cut well into the dewatering box, and dehydrated in 75\% alcohol for $4 \mathrm{~h}, 85 \%$ alcohol, and 90\% alcohol for 2 hours each; anhydrous ethanol I and anhydrous ethanol II for $30 \mathrm{~min}$; alcohol benzene, xylene I, and xylene II for 5-10 min; and wax I, wax II, and wax III for $1 \mathrm{~h}$. The tissue was soaked in wax and embedded. The paraffin tissues were cut into $4 \mu \mathrm{m}$ thick sections with a cryotome and were stained with hematoxylin and eosin (H\&E), periodic acid-Schiff (PAS), or Masson's trichrome $(\mathrm{M}-\mathrm{T})$. The morphological changes in the stained tissues were observed under a light microscope.

2.7. Gut Microbiota Analysis. The feces samples collected from all groups on the third week were used for the microbial community analysis. Total genomic DNAs from the feces of mice were extracted using the E.Z.N.A. ${ }^{\circledR}$ stool DNAKit (Omega Bio-Tek, Norcross, GA, U.S.) according to the manufacturer's instruction. The quality of extracted DNA was checked by $1 \%$ agarose gel electrophoresis and spectrophotometry (optical density at the $260 \mathrm{~nm} / 280 \mathrm{~nm}$ ratio). All extracted DNA samples were stored at $-20^{\circ} \mathrm{C}$ for further analysis.

The V3-V4 hypervariable regions of the 16s rRNA gene were subjected to high-throughput sequencing by Beijing Allwegene Tech, Ltd. (Beijing, China) using the Illumina Miseq PE300 sequencing platform (Illumina, Inc., CA, USA). The V3-V4 regions of the bacterial 16s rRNA gene were amplified with the universal primers of the forward $338 \mathrm{~F}$ (5'-ACTCCTACGGGAGGCAGCAG-3) 5and the reverse $806 \mathrm{R}$ ( $5^{\prime}$-GACTACHVGGGTWTCTAAT- $\left.{ }^{\prime}\right)$. The PCR program was as follows: $95^{\circ} \mathrm{C}$ for $5 \mathrm{~min}$ and 25 cycles at $95^{\circ} \mathrm{C}$ for $30 \mathrm{~s}, 55^{\circ} \mathrm{C}$ for $30 \mathrm{~s}$, and $72^{\circ} \mathrm{C}$ for $30 \mathrm{~s}$ with the final extension of $72^{\circ} \mathrm{C}$ for $10 \mathrm{~min}$. PCR reactions were performed in triplicate: $25 \mu \mathrm{l}$ mixture containing $2.5 \mu \mathrm{l}$ of $10 \times$ Pyrobest Buffer, $2 \mu \mathrm{l}$ of $2.5 \mathrm{mM}$ dNTPs, $1 \mu \mathrm{l}$ of each primer $(10 \mu \mathrm{M})$, $0.4 \mathrm{U}$ of Pyrobest DNA Polymerase (TaKaRa), and $15 \mathrm{ng}$ of template DNA. The amplicon mixture was applied to the MiSeq Genome Sequencer (Illumina, San Diego, CA, USA).

2.8. Illumina MiSeq Sequencing. Amplicons were extracted from 2\% agarose gels and purified using the AxyPrep DNA Gel Extraction Kit (Axygen Biosciences, Union City, CA, U.S.) according to the manufacturer's instructions and quantified using QuantiFluor ${ }^{\mathrm{TM}}$-ST (Promega, U.S.). Purified amplicons were pooled in equimolar and paired-end sequenced $(2 \times 300)$ on an Illumina MiSeq platform according to the standard protocols.

The extraction of high-quality sequences was firstly performed with the QIIME package (Quantitative Insights Into Microbial Ecology) (v1.2.1). Raw sequences were selected 
TABLE 4: Species at different groups of genus classification levels (\%).

\begin{tabular}{|c|c|c|c|c|c|}
\hline Genus & GC & $\mathrm{MC}$ & TJG & HFYG & YXZ \\
\hline Collinsella & $0.01 \%$ & $0.55 \%^{* *}$ & $0.07 \%{ }^{\# \#}$ & $0.64 \%$ & $0.74 \%$ \\
\hline Phascolarctobacterium & $0.00 \%$ & $0.65 \% *$ & $0.08 \%{ }^{\# \#}$ & $0.19 \%$ & $0.17 \%$ \\
\hline Bifidobacterium & $3.10 \%$ & $1.14 \%$ & $1.20 \%$ & $1.80 \%$ & $1.16 \%$ \\
\hline Akkermansia & $0.21 \%$ & $0.37 \%$ & $0.36 \%$ & $0.64 \%$ & $1.09 \%$ \\
\hline Ruminococcaceae_UCG-014 & $3.32 \%$ & $0.95 \% *$ & $2.49 \%$ & $3.35 \%^{\# \#}$ & $2.09 \%$ \\
\hline Anaerostipes & $0.00 \%$ & $0.33 \%$ & $0.09 \%$ & $1.27 \%$ & $1.21 \%{ }^{\# \#}$ \\
\hline Acinetobacter & $0.00 \%$ & $0.11 \% *$ & $0.73 \%$ & $0.03 \%$ & $0.30 \%$ \\
\hline Ruminococcaceae_UCG-013 & $0.87 \%$ & $0.13 \% *$ & $1.15 \%$ & $0.55 \%$ & $0.78 \%{ }^{\#}$ \\
\hline Ruminococcus_torques_group & $0.00 \%$ & $0.42 \% *$ & $0.19 \%$ & $0.11 \%$ & $0.74 \%$ \\
\hline Escherichia-Shigella & $0.00 \%$ & $2.43 \%^{* *}$ & $1.71 \%$ & $2.16 \%$ & $2.31 \%$ \\
\hline Aerococcus & $1.36 \%$ & $0.85 \%$ & $1.55 \%$ & $0.21 \%^{\#}$ & $0.23 \%^{\#}$ \\
\hline Anaerotruncus & $0.01 \%$ & $0.54 \%$ & $0.19 \%$ & $0.37 \%$ & $0.15 \%$ \\
\hline Bacteroides & $0.00 \%$ & $0.20 \%^{* *}$ & $0.07 \%{ }^{\#}$ & $0.78 \%$ & $0.25 \%$ \\
\hline Faecalibaculum & $0.42 \%$ & $0.02 \%{ }^{* *}$ & $0.17 \%$ & $0.13 \%$ & $0.05 \%$ \\
\hline Butyricicoccus & $0.01 \%$ & $1.80 \% *$ & $0.79 \%$ & $0.12 \%^{\#}$ & $0.53 \%$ \\
\hline Staphylococcus & $0.83 \%$ & $0.44 \%$ & $0.19 \%$ & $0.02 \%^{\#}$ & $0.10 \%$ \\
\hline Desulfovibrio & $0.49 \%$ & $0.22 \%$ & $0.34 \%$ & $0.11 \%$ & $0.06 \%$ \\
\hline Jeotgalicoccus & $1.10 \%$ & $2.79 \%$ & $1.72 \%$ & $0.53 \%^{\#}$ & $0.77 \%{ }^{\#}$ \\
\hline Eubacterium_coprostanoligenes_group & $0.23 \%$ & $0.11 \%$ & $0.52 \%$ & $0.67 \%$ & $0.65 \%$ \\
\hline Romboutsia & $10.56 \%$ & $8.65 \%$ & $6.03 \%{ }^{\#}$ & $3.83 \%{ }^{\# \#}$ & $6.92 \%$ \\
\hline Blautia & $0.03 \%$ & $2.52 \% *$ & $2.23 \%{ }^{\# \#}$ & $1.15 \%$ & $2.48 \%$ \\
\hline Subdoligranulum & $0.00 \%$ & $0.62 \% *$ & $2.91 \%{ }^{\#}$ & $0.27 \%$ & $0.66 \%$ \\
\hline Lachnospiraceae_NK4A136_group & $0.27 \%$ & $0.09 \%$ & $1.00 \%$ & $0.05 \%$ & $0.06 \%$ \\
\hline Lactobacillus & $40.83 \%$ & $44.49 \%$ & $37.52 \%$ & $53.23 \%$ & $52.16 \%$ \\
\hline Candidatus_Saccharimonas & $2.28 \%$ & $0.00 \% * *$ & $0.05 \%$ & $0.07 \%^{\#}$ & $0.12 \%$ \\
\hline Oligella & $0.52 \%$ & $0.20 \%$ & $1.44 \%{ }^{\#}$ & $0.10 \%$ & $0.37 \%$ \\
\hline Allobaculum & $6.17 \%$ & $7.75 \%$ & $8.52 \%$ & $4.09 \%$ & $3.27 \%$ \\
\hline Enterococcus & $0.23 \%$ & $4.47 \%$ & $4.30 \%$ & $2.21 \%$ & $2.42 \%$ \\
\hline Facklamia & $0.48 \%$ & $0.43 \%$ & $0.85 \%{ }^{\#}$ & $0.10 \%{ }^{\#}$ & $0.25 \%^{\#}$ \\
\hline Ruminococcus_2 & $0.05 \%$ & $0.11 \%$ & $0.15 \%$ & $0.91 \%^{\#}$ & $0.79 \%{ }^{\#}$ \\
\hline Adlercreutzia & $0.93 \%$ & $0.22 \%$ & $0.13 \%$ & $0.12 \%$ & $0.17 \%$ \\
\hline Enterorhabdus & $1.55 \%$ & $0.27 \% *$ & $0.54 \%^{\#}$ & $0.42 \%$ & $0.48 \%{ }^{\#}$ \\
\hline Helicobacter & $0.00 \%$ & $1.25 \% * *$ & $0.97 \%$ & $1.09 \%$ & $0.56 \%$ \\
\hline Eubacterium_hallii_group & $0.00 \%$ & $0.34 \% *$ & $0.51 \%$ & $0.08 \%$ & $0.31 \%$ \\
\hline Parasutterella & $0.00 \%$ & $0.74 \%$ & $0.15 \%$ & $0.42 \%$ & $0.13 \%$ \\
\hline Corynebacterium_1 & $6.67 \%$ & $5.78 \%$ & $6.60 \%$ & $2.95 \%$ & $3.19 \%$ \\
\hline Ruminococcaceae_UCG-005 & $0.08 \%$ & $0.17 \%$ & $2.22 \%$ & $1.64 \%$ & $1.74 \%$ \\
\hline Clostridium_sensu_stricto_1 & $7.27 \%$ & $0.07 \%$ & $0.01 \%$ & $1.97 \%{ }^{\#}$ & $0.67 \%^{\#}$ \\
\hline Other & $10.08 \%$ & $7.77 \%$ & $9.66 \%$ & $11.04 \%$ & $9.24 \%$ \\
\hline
\end{tabular}

Note: CG: control group; MG: model group; TG: soup group; HG: oil group; YXG: positive group. Compared with the control group, ${ }^{*} p<0.05,{ }^{* *} p<0.01$; compared with the model group, ${ }^{\#} p<0.05,{ }^{\# \#} p<0.01$.

based on sequence length, quality, primer, and tag. The raw sequences were selected, and the low-quality sequences were removed; specific information is as follows: (i) the $300 \mathrm{bp}$ reads were truncated at any site receiving an average quality score $<20$ over a $50 \mathrm{bp}$ sliding window, discarding the truncated reads that were shorter than 50 bp. (ii) Exact barcode matching, 2 nucleotide mismatches in primer matching, and reads containing ambiguous characters were removed. (iii) Only sequences that overlap longer than $10 \mathrm{bp}$ were assembled according to their overlap sequence. Reads which could not be assembled were discarded. The unique sequence set was classified into operational 


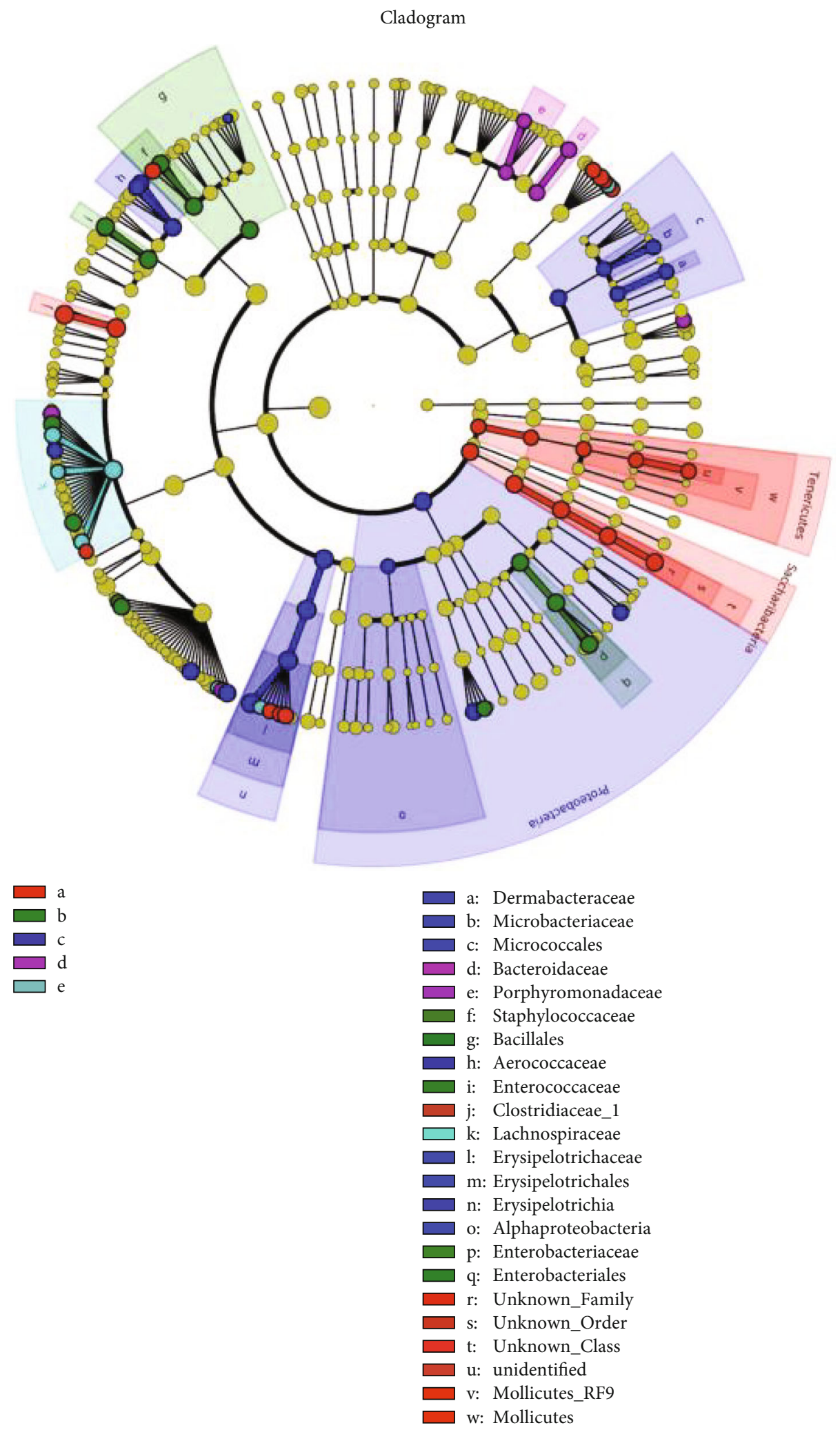

FIGURE 8: Cladogram: analysis of significant difference between samples: a-CG group, b-MG group, c-TG group, d-HG group, and e-YXG group.

taxonomic units (OTUs) under the threshold of $97 \%$ identity using UCLUST. Chimeric sequences were identified and removed using USEARCH (version 8.0.1623). The tax- onomy of each $16 \mathrm{~S}$ rRNA gene sequence was analyzed by UCLUST against the Silva119 16S rRNA database using confidence threshold of $90 \%$. 


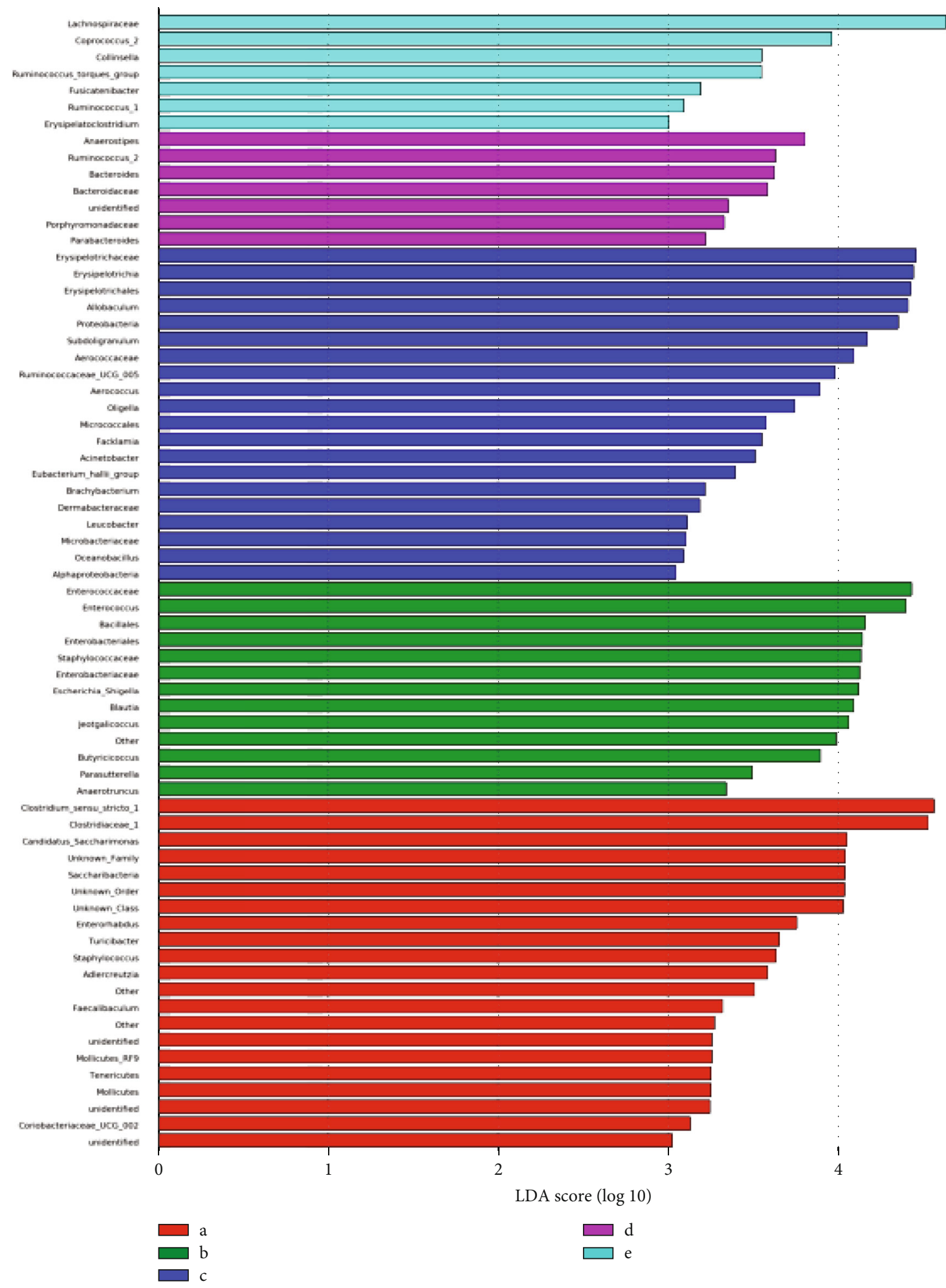

FIGURE 9: LDA score of significantly different bacteria in each group: a-CG group, b-MG group, c-TG group, d-HG group, and e-YXG group. 
2.9. Statistical Analysis. Statistical analysis was performed using SPSS 24.0. The graphs were made using GraphPad Prism 6.0. One-way analysis of variance (ANOVA) and two-tail Student's $t$-test were conducted for comparisons among the multiple groups. $p<0.05$ was considered to be statistically significant.

\section{Results}

3.1. Macro Characterization. Throughout the experiment, the physiological status between the CG group and the other four groups (MG, TG, HG, and YXG group) is significant. The CG group displayed flexible reaction, smooth coat, controlled food intake, and granular stool. The spleen deficiency model in the other four groups (MG, TG, HG, and YXG group) was developed using Xiaochengqi decoction as previously described. The rats in other groups began to have diarrhea on the $3^{\text {rd }}$ day after the establishment of the model. The water content in their stools increased markedly. On the $7^{\text {th }}$ day, the diarrhea was more obvious. The stools were thin, and there was dirt around the anal area. They squinted visually, crouched obviously, piled up, arched back, were tired and sleepy, fed less, reacted slowly, and defecated many times, and their fur was dark and glossy (Figure 1).

3.2. Effect of Caoguo-4 Decoction Volatile Oil on Serum DXylose and AMS Content and Plasma MTL Content in Spleen Deficiency Rats. Table 1 shows that the plasma MTL content in the MC group was higher than that in the CG group $(p<0.05)$. The plasma MTL content in the TG and MG groups was lower than that in the MG group $(p<0.05$ ). The serum D-xylose and AMS content in the MG group was lower than that in the CG group $(p<0.05)$, but for the TG, MG, and YXG groups, serum was significantly increased in the serum D-xylose and AMS levels $(p<0.01$ or $p<0.05)$. This result indicated that Caoguo- 4 decoction and Caoguo- 4 decoction volatile oil could upregulate the content of Dxylose, AML, and MTL in rats.

3.3. The Histopathological Changes in the Intestinal Mucosa. The histopathological changes of the intestinal mucosa of mice in all groups were analyzed by $\mathrm{HE}$ staining (Figure 2). Mice in the CG group showed integrity of the intestinal epithelium and no inflammatory cell infiltration in interstitial cells. But in the MC group, mucosal lesions with a massively destructed epithelium and a mass of glands were decreased with disorder and inflammatory cell infiltration. In the TG and HG group, with a little inflammatory cell infiltration, the structure of the epithelium in the villi of the small intestine was almost complete, only partially broken. This result indicated that Caoguo-4 decoction and Caoguo4 decoction volatile oil could significantly protect intestinal mucosa structure and reduce histologic inflammation.

3.4. OTU Analysis. Figure 3 shows the operational taxonomic unit (OTU) number of all the bacteria in the gut. When compared with the CG group, the MG group had 224 identical species, the TG group had 315 identical species, the HG group had 316 identical species, and the TYG group had 270 identical species. Further, when compared with the
MG group, the TG group had 314 identical species, the HG group had 311 identical species, and the YXG group had 308 identical species. Between the TG group and the HG group, there were 389 identical species. It can be seen that after the treatment using the Caoguo-4 decoction volatile oil, the number of bacterial OTUs in the HG group is the same as that in the TG group; further, there was similar number of bacterial OTUs in the TG group and the CG group. The results indicate that the simultaneous use of the Caoguo-4 decoction volatile oil and the Caoguo- 4 decoction can effectively restore intestinal microbiota and restore intestinal microecological environment.

In Figure 4, we find that the YXG group points are more dispersed, indicating that the intestinal microbial community in this group of rats is the most different. However, the TG group and the HG group points are relatively concentrated, indicating that the difference between the two groups of rat's intestinal microbial community is small. Interestingly, in the HG group, the difference was minimal.

3.5. Alpha Diversity Analysis. The good coverage value for each group was greater than 0.99 , indicating that the results of this sequencing can reflect the actual situation of microorganisms in the sample. The results of alpha diversity analysis between groups (Figure 5) indicated that compared with the CG group, the intestinal microbiota richness index (Figure 5, chaol and observed species) (Table 2) in the MG group was significantly reduced $(p<0.01)$ and the diversity index (PD whole tree) significantly decreased $(p<0.05)$. Further, compared with the MG group, the intestinal microbiota richness index (Figure 5, chaol and observed species) of rats in the TG group and HG group was significantly increased $(p<0.01)$ and the diversity index (whole tree) increased significantly $(p<0.05)$. There was no significant difference in the YXG group $(p>0.05)$. Further, there was no significant difference in the Shannon index (Figure 5, Shannon) in each experimental group $(p>0.05)$, which indicated that the intestinal microbiota of spleen deficiency diarrhea rats had been well recovered posttreatment with Caoguo-4 decoction.

3.6. Species Annotation Analysis. According to the OTUs obtained from the sample sequencing and the species represented by OTUs, the corresponding columnar diagrams of the species were drawn for each sample (Figure 6) at the phylum and belonging to the classification grade.

The distribution of phylum horizontal species is shown in Table 3. The results show that the intestinal structure of rats at the phylum level is mainly thick-walled fungus, Actinomycetes phylum, and deformed fungus phylum, in which the proportion of deformed bacteria phylum is the lowest, the proportion of thick-walled fungus is the highest, and Actinomycetes phylum is the second most dominant flora. Compared to the control group, in the model group, Saccharibacteria $(p<0.05)$, Tenericutes $(p<0.01)$, Euryarchaeota $(p<0.05)$, and Cyanobacteria significantly decreased $(p<0.01)$. However, Proteobacteria significantly increased $(p<0.01)$. Compared with the model group, the Saccharibacteria of the decoction group increased significantly $(p<0.05)$. And Proteobacteria of the volatile oil group 
decreased significantly $(p<0.05)$, Saccharibacteria significantly increased $(p<0.05)$, and there was no significant difference in the positive group.

Figure 7 and Table 4 show the distribution of horizontal species; the results show that when compared with the control group, in the model group, Enterorhabdus $(p<0.05)$, Candidatus_Saccharimonas $(p<0.01)$, and Ruminococcaceae_UCG-013/UCG-014 $(p<0.05)$ significantly decreased. However, Escherichia-Shigella and Helicobacter significantly increased $(p<0.01)$. Compared with the model group, the decoction group had significantly decreased Romboutsia $(p<0.05)$ and significantly increased Subdoligranulum $(p<0.05)$, Enterorhabdus $(p<0.05)$, and Oligella $(p<0.05)$. In the volatile oil group, Candidatus_Saccharimonas $(p<0.05)$ and Ruminococcaceae_UCG-014 $(p<0.01)$ significantly increased and Aerococcus $(p<0.05)$, Butyricicoccus $(p<0.05)$, and Romboutsia $(p<0.01)$ significantly decreased. In the positive group, Aerococcus $(p<0.01)$, Ruminococcaceae_UCG-013 $(p<0.01)$, and Jeotgalicoccus $(p<0.01)$ significantly decreased, whereas Anaerostipes $(p<0.01)$ and Enterorhabdus $(p<0.05)$ significantly increased.

3.7. Analysis of Significant Difference between Samples. The circle radiating from the inside to the outside represents the classification level from the door to the genus (or species). Each small circle at different levels represents a classification at this level, and the diameter size of the small circle is proportional to the relative abundance size of each genus or species (Figure 8). With coloring principle, the species without significant difference is colored yellow; the different species biomarkers follow the group coloring: the red node represents the microbial group that plays an important role in the "red group" and the green node represents the microbial group that plays an important role in the "green group." The name of the species is represented in the English alphabet in the figure and is shown in the legend on the right.

Figure 9 shows the species in which the linear discriminant analysis (LDA) score is greater than the set value, that is, the biomarker with statistically significant differences. This figure indicates species with significant differences in abundance in different groups, and the length of the histogram represents the size of the significantly different species. The longer the column, the greater the LDA value. Clostridium, Clostridiaceae, Enterorhabdus, Staphylococcus, soft-wall fungus door Tenericutes, and Coriobacteriaceae had the highest abundance in the control group. Enterococcaceae, Bacillales, Enterobacteriales, Escherichia, Blautia, and the genus Anaerotruncus were the most abundant in the model group. Erysipelotrichia, Erysipelotrichaeae, Erysipelotrichichales, Allobaculum, Proteobacteria, Aerococcaceae, Ruminococcaceae, Aerococcus, Oligella, Micrococcales, Eubacterium Hallii, Brachybacterium, Dermabacteraceae, Microbacteriaceae, marine Oceanobacillus, and $\alpha$-Proteobacteria had the highest abundance in the decoction group. Anaerostipes, Ruminococcus 2, Bacteroides, Bacteroidaceae, Porphyromonadaceae, and Parabacteroides had the highest abundance in volatile oil groups. The different species with the highest abundance in the positive group were Lachnospiraceae, Coprococcus, and Ruminococcaceae.

\section{Discussion}

To test our hypothesis that regulation of intestinal microbiota by the Caoguo-4 decoction volatile oil is related to its antidiarrheal efficacy, we established the SDD model in rats demonstrated by indices of diarrhea and clinical symptoms, which is consistent with previous studies. Its main function is to affect the power of the gastrointestinal tract and can stimulate the secretion of pepsin and pancreatic fluid along with gallbladder contraction. The effect on gastrointestinal movement is manifested in increasing the tension of the lower esophageal sphincter, promoting the movement of the stomach, small intestine, and gallbladder, and contributing to the absorption of food. The experimental results showed that the plasma motilin in the model group was significantly higher than that in the control group, which was consistent with the previous studies such as $\mathrm{Lu}$ [16] and $\mathrm{Li}$ and Zhen [17], but the results are inconsistent with studies from groups such as Song et al. [18] and Shao et al. [19]; hence, there is a need to further study its mechanism. After administration with either the decoction group or volatile oil group, there is significant decrease in motilin, and the decrease in the volatile oil group was more obvious thus proving that this group was most efficient for the treatment of SDD. Results also showed that there was some damage to the intestinal mucosal structure in the model group, and the Caoguo-4 decoction and the Caoguo-4 decoction volatile oil group could improve the damaged epithelial structure and reduce inflammation.

The results of OTU analysis and alpha diversity analysis showed that the intestinal microbiota of spleen deficiency model rats changed significantly. With the increase in the number of samples and their corresponding grade values, the number of bacteria and relative abundance tend to be more towards a certain constant value. This indicates that the sequencing depth is sufficient to reflect the level of community richness, the number of OTUs is close to the actual situation, and the uniformity of community composition is higher. Additionally, the coverage value is the coverage of the sample library in the experiment, with $10 \%$ as the upper limit; the higher the value, the higher the probability of the sequence being detected in the sample, which means that the sequencing results can reflect the real situation of the sample.

Further, the richness and diversity of intestinal microbiota also decreased significantly. Through intervention with decoction and volatile oil, the richness and diversity of intestinal flora recovered significantly, but there was no significant change in the positive group. This shows that the intestinal microbiota in rats with SDD has been destroyed, and both decoction and volatile oil can restore the unbalanced intestinal microbiota, and it can be speculated that decoction and volatile oil are superior to Smecta in the treatment of spleen deficiency diarrhea.

Intestinal microbiota are the most important and diverse microbial community living in the human body [20], and in order to maintain the body function in a stable state, intestinal microbiota will restrict each other to achieve dynamic balance. Intestinal microbiota disorders in the TCM are 
considered important biological basis for "spleen deficiency" [21]. Further, the intestinal microecological changes of the SDD model are closely related to spleen inactivation. In this study, the thick-wall fungus door accounted for a large proportion in the gut, but there was no significant difference between the groups in this experiment. The change of the main intestinal microbiota caused by SDD is the decrease in the number of bacteria and Saccharibacteria in the intestinal tract (Bacteroidetes), but the number of bacteria in the deformed fungus door (Proteobacteria) increased correspondingly. This change was mainly due to the high nutritional requirements of the anaerobic bacteria Gramnegative Bacillus (mostly Bacillus-gate bacteria) caused by intestinal microenvironment destruction and the proliferation of lactobacillus bacteria with strong antibiotic resistance and lower nutritional requirements [22]. In this experiment, the Proteobacteria in the diarrhea model group of SDD was significantly increased, and the treatment of volatile oil in Caoguo-4 decoction inhibited the Proteobacteria in rats, and the bacteria of Bacillus were recovered. In this paper, the number of Mycobacterium doors in the model group was lower than that in the control group, and the number of Bacillus in the volatile oil group was the best to recover.

At the level of diarrhea, the genus Escherichia coli, Enterococcus, and pylori were observed to be increased, but the genus Bifidobacterium and rumen were reduced. Escherichia coli is the main pathogen causing the global epidemic of infectious diarrhea and outbreak of local diarrhea. The genus Enterococcus is a gram-positive coccus, which is aerobic or anaerobic and is in control of the host. Under controlled circumstances, it will not endanger the health of the host, but in the lack of nutrients, high alkaline and other harsh environment for a prolonged time can cause pathological changes, leading to infection disease $[23,24]$. Bifidobacterium is a probiotic in the gut of humans and animals. It participates in a series of physiological processes, such as immunity, nutrition, digestion, and protection, and has the function of maintaining the balance of controlling intestinal microbiota, inhibiting the growth of pathogenic bacteria, and preventing and controlling constipation, dysentery, and gastrointestinal disorders [20]. In this study, each drug group had a conditioning effect on lactic acid bacilli in the gut of rats in the model group.

In addition, the regulation degree of the Caoguo-4 decoction agent and volatile oil on intestinal microbiota of SDD model rats was different. Compared with decoction, volatile oil had a conditioning effect on rumen fungus, pseudo-rod bacteria, and Saccharibacteria. Compared with the volatile oil group, decoction has a conditioning effect on Bacillus, Enterococcus, Romboutsia, Subdoligranulum, Enterorhabdus, and Oligella. The results show that the potential mechanism of the Caoguo- 4 decoction agent and volatile oil on the SDD rat model is different, which may suggest the unique regulation mechanism of volatile oil on intestinal microbiota. In short, the two intervention groups changed the structure of intestinal microbiota and increased the beneficial bacteria such as the Bifidobacterium genus, rumen fungus, Arabidopsis bacteria, and Saccharibacteria and reduced harmful bacteria such as deformed fungus doors, Escherichia coli, Romboutsia, and Blautia. Further, by analyzing the species or communities with significant differences between the groups, it was found that the changes of Enterococcus, Enterobacter, and Escherichia coli may be related to the diarrhea index of spleen deficiency.

\section{Conclusion}

In summary, Caoguo-4 decoction and volatile oils are beneficial against diarrhea, which reduced the incidence of diarrhea and improved stool. Our research proved that Caoguo-4 decoction and volatile oils can upregulate the content of D-xylose, AML, and MTL in rats, changing the structure of intestinal microbiota by increasing the beneficial bacteria such as the Bifidobacterium genus, rumen fungus, Arabidopsis bacteria, and Saccharibacteria and reducing harmful bacteria such as deformed fungus doors, Escherichia coli, Romboutsia, and Blautia. To achieve treatment of spleen deficiency diarrhea with its mechanism being still unknown, further research is needed.

\section{Data Availability}

All data used to support the findings of this study are available from the corresponding author upon request.

\section{Ethical Approval}

The animal study was reviewed and approved by the Animal Ethics Committee of Jiangxi University of Traditional Chinese Medicine.

\section{Conflicts of Interest}

The authors declare that there is no conflict of interests regarding the publication of this paper.

\section{Authors' Contributions}

MY and FW cosupervised the study. LM, XFZ, and FW codesigned the study based on the sequenced data. FW, YZG, and QYC codesigned and implemented the therapeutic measures and sampling. LFW, ZYL, and FQL analyzed the data and made the plots and tables of the result. LM, YM, and FW wrote and revised the manuscript, and all authors proofread and improved the manuscript. Fang Wang is the co-first author.

\section{Acknowledgments}

This study was supported by grants from the Project of National Natural Science Foundation of China (81960714), the Jiangxi Province Science and Technology Major Projects (20194ABC28009), the funding from Jiangxi University of Traditional Chinese Medicine (JXSYLXK-ZHYAO083, JXSYLXKZHYAO084), the "Double First-Rate" Construction Program of the Inner Mongolia University for Nationalities (NMDGJ0012), and the Natural Science Foundation of Inner Mongolia Autonomous Region of China (2020BS08008). 


\section{References}

[1] J. Wang, W. Feng, S. Zhang et al., "Ameliorative effect of Atractylodes macrocephala essential oil combined with Panax ginseng total saponins on 5-fluorouracil induced diarrhea is associated with gut microbial modulation," Journal of Ethnopharmacology, vol. 238, article 111887, 2019.

[2] Q. Liu and C. J. Wang, "Treatment of chronic diarrhea due to intestinal microbiota imbalance according to the theory of "spleen deficiency"," Clinical Journal of Traditional Chinese Medicine, vol. 29, no. 12, pp. 2032-2033, 2017.

[3] W. Ruan, Z. H. W. Chen, and J. T. Yan, "“Four-step massage” in treating childhood spleen-deficiency diarrhea," Journal of Acupuncture and Tuina Science, vol. 4, no. 5, pp. 271-273, 2006.

[4] C. A. Lozupone, J. I. Stombaugh, J. I. Gordon, J. K. Jansson, and R. Knight, "Diversity, stability and resilience of the human gut microbiota," Nature, vol. 489, no. 7415, pp. 220-230, 2012.

[5] B. Y. Xu, Y. Q. Yan, J. C. Huang, B. Q. Yin, Y. X. Pan, and L. B. Ma, "Cortex Phellodendri extract santi-diarrhea effect in mice related to its modification of gut microbiota," Biomedicine \& Pharmacotherapy, vol. 3, no. 123, pp. 63-63, 2020.

[6] G. Solano-Aguilar, K. Fernandez, H. Ets et al., "Characterization of fecal microbiota of children with diarrhea in 2 locations in Colombia," Journal of Pediatric Gastroenterology and Nutrition, vol. 56, no. 5, pp. 503-511, 2013.

[7] J. Ma, Z. G. Yang, J. C. Wei et al., "Effects of different processed products of Radix Codonopsis on intestinal microbiota of rats with spleen deficiency," Medicinal Plant, vol. 11, no. 1, pp. 6363, 2020.

[8] R. Wang, Y. Shi, X. Xie et al., "Use of Shenhuang paste on Shenque point improves chemotherapy induced gastrointestinal toxicity in breast cancer: a protocol for randomized controlled trial," Medicine, vol. 100, no. 15, article e25097, 2021.

[9] W. B. Han, "Clinical observation of acupoint application for slow transit constipation due spleen deficiency and intestine dryness," Shanghai Journal of Acupuncture and Moxibustion, vol. 37, no. 12, pp. 1359-1362, 2018.

[10] K. X. Guo, M. J. Peng, X. X. Peng, H. Y. Hui, and Z. J. Tan, "Effects of Qiweibaizhu powder on the intestinal bacterial diversity in dysbacteriotic diarrhea mice," Wei Sheng Wu Hsueh T'ung Pao Microbiology, vol. 45, no. 7, pp. 1470-1479, 2018.

[11] Inner Mongolia Autonomous Region Hospital, Mongolian Medicine Verification Prescription (Mongolian Version), Ancient Xinhua Printing of Inner Mongolia, Hohhot, Mongolia, 1971.

[12] The Editorial Board of Chinese Medical Encyclopedia, Chinese Medical Encyclopedia (Mongolian Edition), Shanghai Science and Technology Press, Shanghai, China, 1992.

[13] Y. Q. Wang, Y. Z. Yang, Z. F. Wang, Y. K. Xiong, and M. Yang, "Traditional function and modern research progress on volatile oil in Chinese materia medica," Chinese Traditional and Herbal Drugs, vol. 49, no. 2, pp. 455-457, 2018.

[14] Y. H. Li, D. M. Zhang, S. Y. Wang et al., "Development of animal model of spleen deficiency syndrome," Guiding Journal Of Traditional Chinese Medicine, vol. 25, no. 1, pp. 100-103, 2019.

[15] W. Zhang, H. Y. Zhang, and S. P. Huang, "The study of composite factors make spleen-deficient model in rats," Lishizhen Medicine and Materia Medica Research, vol. 26, no. 4, pp. 1022-1023, 2015.
[16] Z. Lu, Mouse Gut Microbiota Composition and Metabolites Change Caused by Inulin Addition in High Fat Diet, Tsinghua University, 2017.

[17] B. F. Li and C. H. Zhen, "The influence of Smeta on serum motilin and gastrin levels in rats with sp," Zhejiang Clinical Medical Journal, vol. 10, pp. 841-843, 2004.

[18] Y. P. Song, H. F. Chen, Y. X. Hu, S. S. Tan, X. Q. Luo, and W. L. Yang, "Effect of Pericarpium Citri Reticulatae and its main active ingredients on gastrin in serum, acetylcholine, motilin, substance $P$ and vasoactive intestinal peptide in plasma of rats," Pharmacology and Clinics of Chinese Materia Medice, vol. 33, no. 3, 2017.

[19] N. J. Shao, X. Ma, X. X. Zhu, H. D. Wang, and Q. Gao, "Effects of Yunpiwenyang granule on MTL, SS and VIP in the blood of mouse with splenic deficiency diarrhea," Journal of Changchun University of Traditional Chinese Medicine, vol. 30, no. 6, 2014.

[20] Y. Y. Chen, R. Wang, J. H. Zhang et al., "Effects of intestinal microflora on drug metabolism," Chinese Pharmacological Bulletin, vol. 35, no. 2, pp. 168-175, 2019.

[21] G. Yuan and S. Qinglong, "Advances in pharmacology research of Shenqi Baizhu powder based on intestinal microecology," Lishizhen Medicine and Materia Medica Research, vol. 29, no. 3, 2018.

[22] H. Yao, Z. Lv, H. F. Xu, and J. R. Su, "The study of regulation of berberine to gut microflora in mouse model with Clostridium difficile associated diarrhea," The Chinese Medical Journal, vol. 53, no. 1, pp. 77-77, 2018.

[23] V. Shetty, S. H. Kumar, A. K. Shetty, I. Karunasagar, and I. Karunasagar, "Prevalence and characterization of diarrheagenic Escherichia coli isolated from adults and children in Mangalore, India," Journal of Laboratory Physicians, vol. 4, no. 1, pp. 24-029, 2012.

[24] S. Patzi-Vargas, M. B. Zaidi, Perez-Martinez et al., "Diarrheagenic Escherichia coli carrying supplementary virulence genes are an important cause of moderate to severe diarrhoeal disease in Mexico," PLoS Neglected Tropical Diseases, vol. 9, no. 3,2015 . 